\title{
Thermophysical properties of glycols and glymes
}

Pedro J. Carvalho ${ }^{a}$, Cristofe H. G. Fonseca ${ }^{\mathrm{a}}$, Maria-Luísa C. J. Moita ${ }^{\mathrm{b}}$, Ângela F. S. Santos ${ }^{\mathrm{c}}$ and João A. P. Coutinho ${ }^{a, *}$

${ }^{\text {a }}$ CICECO - Aveiro Institute of Materials, Department of Chemistry, University of Aveiro, 3810-193 Aveiro, Portugal

${ }^{b}$ Departamento de Química e Bioquímica, Centro de Química e Bioquímica, Faculdade de Ciências, Universidade de Lisboa, 1749-016 Lisboa, Portugal

'Departamento de Química e Bioquímica, Centro de Química Estrutural, Faculdade de Ciências, Universidade de Lisboa, 1749-016 Lisboa, Portugal

*Corresponding author. Tel.: +351 234401 507; fax: +351 234370084

E-mail address: jcoutinho@ua.pt 


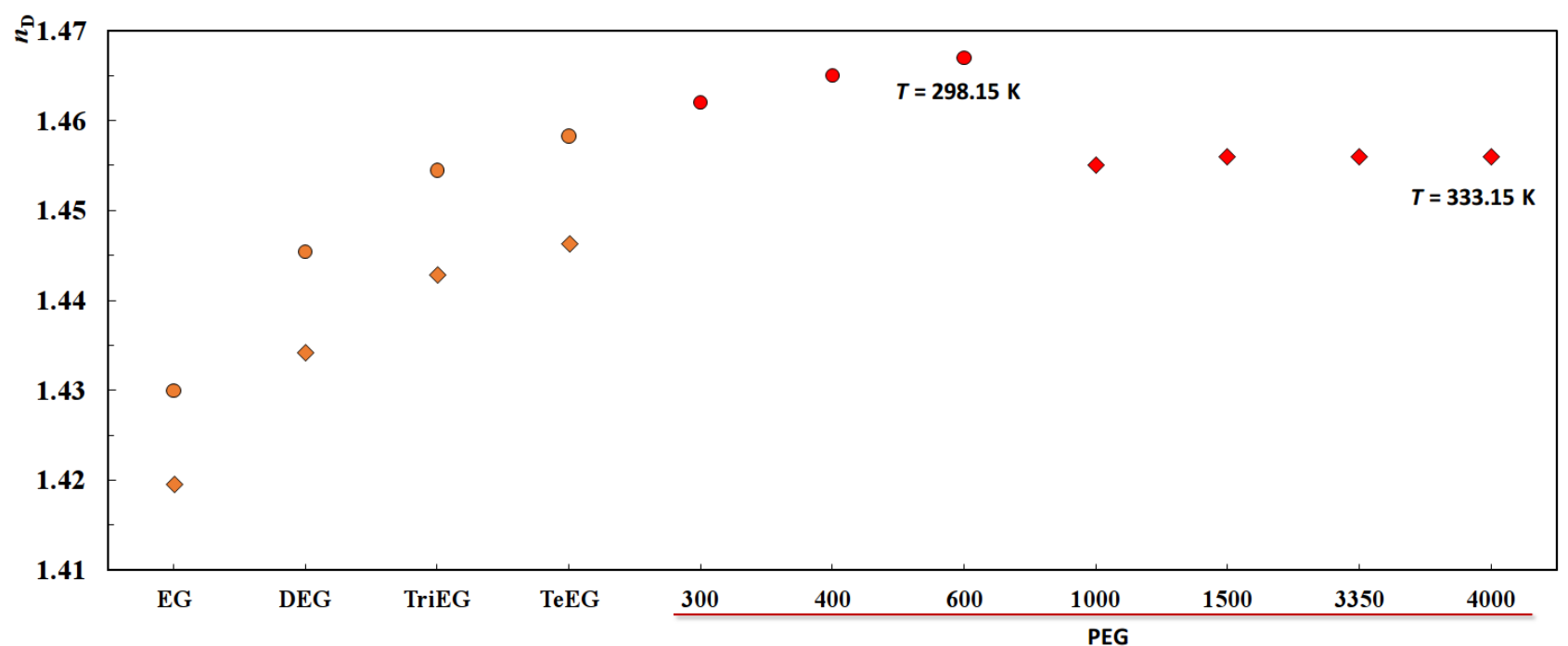

Figure S1. Refractive index as function of the mono, di, tri and tetra ethylene glycol and different molecular weight PEGs. Filled circles and diamond symbols represent the refractive indices at $298.15 \mathrm{~K}$ and $333.15 \mathrm{~K}$, respectively.

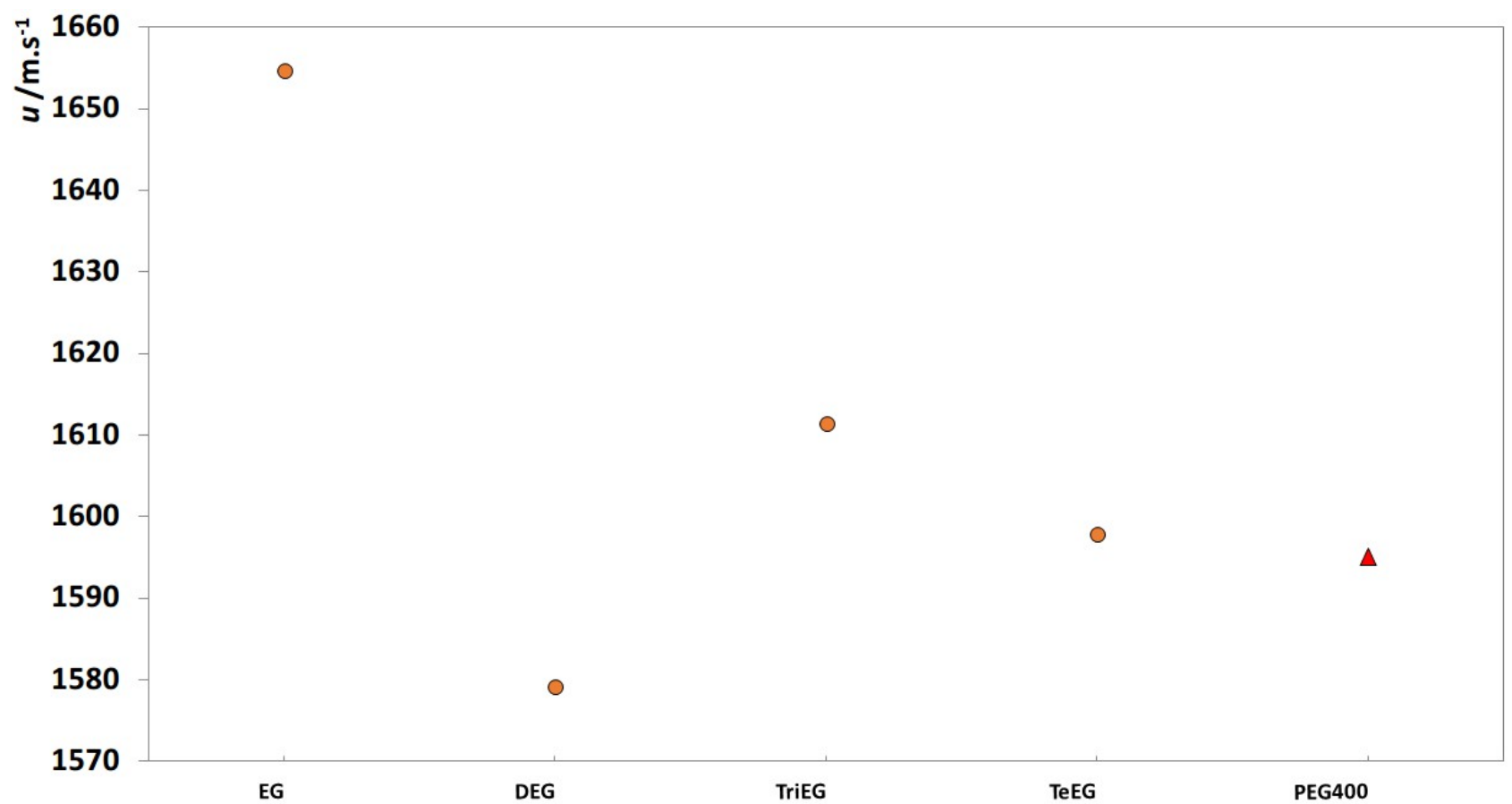

Figure S2. Sound speed for the mono, di, tri and tetra ethylene glycol (orange circles) and PEG400 (red triangle), at $298.15 \mathrm{~K}$. 
Table S1. Experimental density data for the glycols and glymes aqueous solutions as function of the temperature and compound's mole fraction.

\begin{tabular}{|c|c|c|c|c|c|c|c|c|c|c|}
\hline \multirow{4}{*}{$T / \mathbf{K}$} & \multicolumn{10}{|c|}{$\rho / \mathrm{kg} \cdot \mathrm{m}^{-3}$} \\
\hline & \multicolumn{5}{|c|}{ DEG } & \multicolumn{5}{|c|}{ DEGEE } \\
\hline & & & & & & & & & & \\
\hline & 0.9070 & 0.9589 & 0.9698 & 0.9900 & 0.9901 & 0.9674 & 0.9740 & 0.9826 & 0.9887 & 0.9899 \\
\hline 283.15 & 1123.135 & 1123.266 & 1123.273 & 1123.325 & 1123.327 & 997.734 & 997.587 & 997.387 & 997.339 & 997.227 \\
\hline 288.15 & 1119.575 & 1119.710 & 1119.716 & 1119.767 & 1119.771 & 993.310 & 993.160 & 992.963 & 992.908 & 992.793 \\
\hline 293.15 & 1116.018 & 1116.151 & 1116.159 & 1116.211 & 1116.213 & 988.877 & 988.725 & 988.528 & 988.475 & 988.357 \\
\hline 298.15 & 1112.454 & 1112.585 & 1112.594 & 1112.649 & 1112.651 & 984.434 & 984.285 & 984.088 & 984.028 & 983.913 \\
\hline 303.15 & 1108.881 & 1109.016 & 1109.025 & 1109.077 & 1109.081 & 979.981 & 979.829 & 979.636 & 979.572 & 979.459 \\
\hline 308.15 & 1105.295 & 1105.431 & 1105.442 & 1105.496 & 1105.498 & 975.514 & 975.363 & 975.164 & 975.102 & 974.991 \\
\hline 313.15 & 1101.695 & 1101.834 & 1101.843 & 1101.900 & 1101.903 & 971.029 & 970.880 & 970.684 & 970.620 & 970.511 \\
\hline 323.15 & 1094.447 & 1094.595 & 1094.603 & 1094.667 & 1094.668 & 962.022 & 961.873 & 961.673 & 961.608 & 961.499 \\
\hline 333.15 & 1087.119 & 1087.278 & 1087.288 & 1087.359 & 1087.356 & 952.945 & 952.794 & 952.591 & 952.534 & 952.422 \\
\hline \multirow[t]{3}{*}{343.15} & 1079.710 & 1079.883 & 1079.897 & 1079.973 & 1079.969 & 943.775 & & 943.425 & & 943.264 \\
\hline & \multicolumn{5}{|c|}{ EGEE } & \multicolumn{5}{|c|}{ DEGME } \\
\hline & 0.9426 & 0.9490 & 0.9549 & 0.9567 & 0.9670 & 0.9483 & 0.9601 & 0.9674 & 0.9893 & 0.9932 \\
\hline 283.15 & 943.675 & 943.444 & 943.150 & 943.112 & 942.599 & 1029.621 & 1029.502 & 1029.287 & 1028.835 & 1028.734 \\
\hline 288.15 & 939.208 & 938.975 & 938.680 & 938.638 & 938.120 & 1025.244 & 1025.129 & 1024.903 & 1024.451 & 1024.350 \\
\hline 293.15 & 934.713 & 934.479 & 934.183 & 934.140 & 933.622 & 1020.866 & 1020.744 & 1020.520 & 1020.059 & 1019.951 \\
\hline 298.15 & 930.190 & 929.953 & 929.657 & 929.614 & 929.099 & 1016.470 & 1016.344 & 1016.121 & 1015.651 & 1015.546 \\
\hline 303.15 & 925.638 & 925.399 & 925.102 & 925.055 & 924.540 & 1012.059 & 1011.934 & 1011.709 & 1011.235 & 1011.124 \\
\hline 308.15 & 921.054 & 920.815 & 920.517 & 920.467 & 919.951 & 1007.635 & 1007.505 & 1007.280 & 1006.801 & 1006.688 \\
\hline 313.15 & 916.435 & 916.194 & 915.892 & 915.843 & 915.328 & 1003.193 & 1003.063 & 1002.834 & 1002.352 & 1002.240 \\
\hline 323.15 & 907.087 & 906.839 & 906.540 & 906.493 & 905.969 & 994.251 & 994.119 & 993.893 & 993.397 & 993.288 \\
\hline 333.15 & 897.580 & 897.329 & 897.036 & 896.985 & 896.461 & 985.224 & 985.089 & 984.870 & 984.365 & 984.259 \\
\hline \multirow[t]{3}{*}{343.15} & 887.911 & 887.628 & 887.337 & 887.297 & 886.774 & 976.029 & 975.972 & 975.754 & 975.241 & 975.146 \\
\hline & \multicolumn{6}{|c|}{ DEGDEE } & & \multicolumn{3}{|c|}{ EG } \\
\hline & 0.9700 & 0.9742 & 0.9778 & 0.9836 & 0.9875 & 0.9911 & & 0.9886 & 0.9943 & 0.9959 \\
\hline 283.15 & 918.572 & 918.371 & 918.271 & 917.783 & 917.643 & 917.567 & & 1119.903 & 1120.066 & 1120.113 \\
\hline 288.15 & 913.742 & 913.543 & 913.443 & 912.955 & 912.820 & 912.740 & & 1116.426 & 1116.590 & 1116.638 \\
\hline 293.15 & 908.910 & 908.705 & 908.610 & 908.126 & 907.993 & 907.914 & & 1112.944 & 1113.108 & 1113.155 \\
\hline 298.15 & 904.074 & 903.873 & 903.775 & 903.300 & 903.166 & 903.090 & & 1109.463 & 1109.613 & 1109.661 \\
\hline 303.15 & 899.235 & 899.035 & 898.937 & 898.467 & 898.334 & 898.256 & & 1105.950 & 1106.110 & 1106.155 \\
\hline 308.15 & 894.389 & 894.189 & 894.092 & 893.628 & 893.496 & 893.419 & & 1102.430 & 1102.586 & 1102.634 \\
\hline 313.15 & 889.537 & 889.339 & 889.241 & 888.783 & 888.652 & 888.577 & & 1098.889 & 1099.049 & 1099.095 \\
\hline 323.15 & 879.812 & 879.619 & 879.518 & 879.074 & 878.945 & 878.873 & & 1091.746 & 1091.903 & 1091.952 \\
\hline 333.15 & 870.056 & 869.867 & 869.760 & 869.332 & 869.203 & 869.134 & & 1084.507 & 1084.669 & 1084.718 \\
\hline 343.15 & 860.264 & 860.076 & 859.966 & 859.550 & 859.411 & 859.346 & & 1077.163 & 1077.326 & 1077.370 \\
\hline
\end{tabular}


Table S2. Experimental sound speed data for the glycos and glymes aqueous solutions as function of the temperature and compound's mole fraction.

$$
u / \mathrm{m} \cdot \mathrm{s}^{-1}
$$

DEG

DEGEE

\begin{tabular}{|c|c|c|c|c|c|c|c|c|c|c|c|}
\hline $\begin{array}{l}T / \mathbf{K} \\
x_{\text {glycol }}\end{array}$ & 0.9070 & 0.9589 & 0.9698 & 0.9900 & 0.9901 & 0.9674 & 0.9740 & 0.9826 & 0.9887 & 0.9899 & \\
\hline 283.15 & 1620.3 & 1617.8 & 1617.4 & 1616.4 & 1616.5 & 1430.8 & 1430.3 & 1429.4 & 1429.6 & 1429.6 & \\
\hline 288.15 & 1608.0 & 1605.5 & 1605.2 & 1604.1 & 1604.2 & 1412.8 & 1412.2 & 1411.4 & 1411.4 & 1411.2 & \\
\hline 293.15 & 1595.9 & 1593.3 & 1593.0 & 1591.9 & 1592.0 & 1394.9 & 1394.3 & 1393.5 & 1393.6 & 1393.3 & \\
\hline 298.15 & 1583.8 & 1581.0 & 1580.8 & 1579.6 & 1579.8 & 1377.3 & 1376.5 & 1375.7 & 1375.7 & 1375.4 & \\
\hline 303.15 & 1571.7 & 1569.0 & 1568.6 & 1567.5 & 1567.4 & 1359.6 & 1358.8 & 1358.0 & 1357.9 & 1357.5 & \\
\hline 308.15 & 1559.6 & 1557.0 & 1556.6 & 1555.4 & 1555.4 & 1342.0 & 1341.3 & 1340.5 & 1340.1 & 1340.0 & \\
\hline 313.15 & 1547.5 & 1545.0 & 1544.6 & 1543.4 & 1543.3 & 1324.4 & 1323.9 & 1323.1 & 1322.7 & 1322.5 & \\
\hline 323.15 & 1523.4 & 1520.9 & 1520.5 & 1519.3 & 1519.2 & 1289.8 & 1289.3 & 1288.5 & 1287.9 & 1287.8 & \\
\hline 333.15 & 1499.0 & 1496.6 & 1496.2 & 1495.1 & 1494.9 & 1255.6 & 1255.0 & 1254.2 & 1253.7 & 1253.5 & \\
\hline 343.15 & 1474.6 & 1472.2 & 1471.8 & 1470.8 & 1470.5 & 1221.7 & 1221.1 & 1220.3 & 1220.0 & 1219.7 & \\
\hline & \multicolumn{5}{|c|}{ EGEE } & \multicolumn{6}{|c|}{ DEGME } \\
\hline & 0.9426 & 0.9490 & 0.9549 & 0.9567 & 0.9670 & 0.9373 & 0.9483 & 0.9601 & 0.9674 & 0.9893 & 0.9932 \\
\hline 283.15 & 1364.0 & 1365.8 & 1366.2 & 1366.8 & 1361.6 & 1473.4 & 1472.6 & 1471.2 & 1470.8 & 1468.4 & 1468.6 \\
\hline 288.15 & 1346.2 & 1347.5 & 1347.9 & 1348.4 & 1343.5 & 1455.8 & 1455.1 & 1453.9 & 1453.3 & 1451.0 & 1451.0 \\
\hline 293.15 & 1328.3 & 1329.2 & 1329.6 & 1330.2 & 1325.6 & 1438.7 & 1438.0 & 1436.7 & 1436.1 & 1433.7 & 1433.4 \\
\hline 298.15 & 1310.5 & 1311.1 & 1311.3 & 1311.8 & 1307.8 & 1421.6 & 1420.8 & 1419.5 & 1418.9 & 1416.4 & 1416.1 \\
\hline 303.15 & 1292.8 & 1293.0 & 1293.2 & 1292.6 & 1289.8 & 1404.5 & 1403.6 & 1402.3 & 1401.7 & 1399.1 & 1398.9 \\
\hline 308.15 & 1275.0 & 1275.3 & 1275.2 & 1274.3 & 1272.0 & 1387.3 & 1386.5 & 1385.1 & 1384.5 & 1381.9 & 1381.6 \\
\hline 313.15 & 1257.4 & 1257.6 & 1256.6 & 1256.1 & 1254.3 & 1370.3 & 1369.4 & 1368.1 & 1367.5 & 1364.9 & 1364.6 \\
\hline 323.15 & 1222.2 & 1222.2 & 1220.3 & 1220.2 & 1218.9 & 1336.5 & 1335.6 & 1334.3 & 1333.7 & 1330.9 & 1330.3 \\
\hline 333.15 & 1187.1 & 1186.9 & 1185.1 & 1184.9 & 1183.7 & 1302.9 & 1302.1 & 1300.7 & 1300.2 & 1297.3 & 1296.7 \\
\hline 343.15 & 1152.0 & 1151.1 & 1150.1 & 1149.8 & 1148.6 & 1269.6 & 1268.8 & 1267.4 & 1266.9 & 1263.9 & 1263.3 \\
\hline
\end{tabular}

DEGDEE EG

$\begin{array}{llllllll}0.9700 & 0.9742 & 0.9778 & 0.9836 & 0.9875 & 0.9911\end{array}$

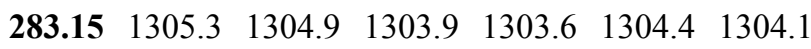

$0.98860 .9943 \quad 0.9959$

$\mathbf{2 8 8 . 1 5} 1284.9 \quad 1284.6 \quad 1283.6 \quad 1283.3 \quad 1284.0 \quad 1284.0$

$1690.2 \quad 1689.0 \quad 1690.6$

$\begin{array}{lllllll}\mathbf{2 9 3 . 1 5} & 1264.8 & 1264.4 & 1263.6 & 1263.2 & 1263.9 & 1263.8\end{array}$

$1678.4 \quad 1678.1 \quad 1678.6$

$\begin{array}{lllllll}298.15 & 1244.9 & 1244.6 & 1243.7 & 1243.4 & 1244.1 & 1244.0\end{array}$

$1666.6 \quad 1666.3 \quad 1666.6$

$\begin{array}{lllllll}303.15 & 1225.2 & 1224.9 & 1224.0 & 1223.6 & 1224.4 & 1224.2\end{array}$

$1654.7 \quad 1654.8 \quad 1654.6$

308.15 $1205.6 \quad 1205.3 \quad 1204.6 \quad 1204.1 \quad 1204.7 \quad 1204.6$

$1643.0 \quad 1643.0 \quad 1642.7$

$\begin{array}{lllllll}313.15 & 1186.4 & 1186.0 & 1185.3 & 1184.7 & 1184.7 & 1185.3\end{array}$

$1631.2 \quad 1631.3 \quad 1630.9$

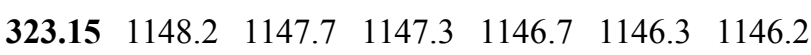

$\begin{array}{lll}1619.5 & 1619.4 & 1619.1\end{array}$

$\begin{array}{llllllll}333.15 & 1111.0 & 1110.4 & 1110.0 & 1109.5 & 1109.1 & 1108.9\end{array}$

$\begin{array}{lll}1595.7 & 1595.5 \quad 1595.2\end{array}$

$\begin{array}{lll}1571.7 & 1571.5 & 1571.2\end{array}$

$\begin{array}{lllllll}\mathbf{3 4 3 . 1 5} & 1074.5 & 1074.0 & 1073.7 & 1073.1 & 1072.6 & 1072.7\end{array}$

$1547.6 \quad 1547.4 \quad 1547.1$

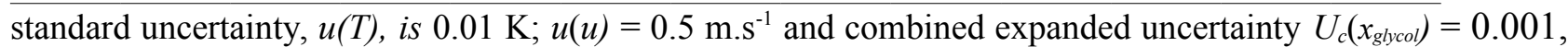
with an approximately $95 \%$ level of confidence. 
Table S3. Least squares fitting coefficients of the compound's aqueous solutions densities, the corresponding uncertainties of the extrapolated densities of the pure glycols and glymes (calculated by error propagation), $u(\rho)$, the number of points used for each fit and the respective composition range.

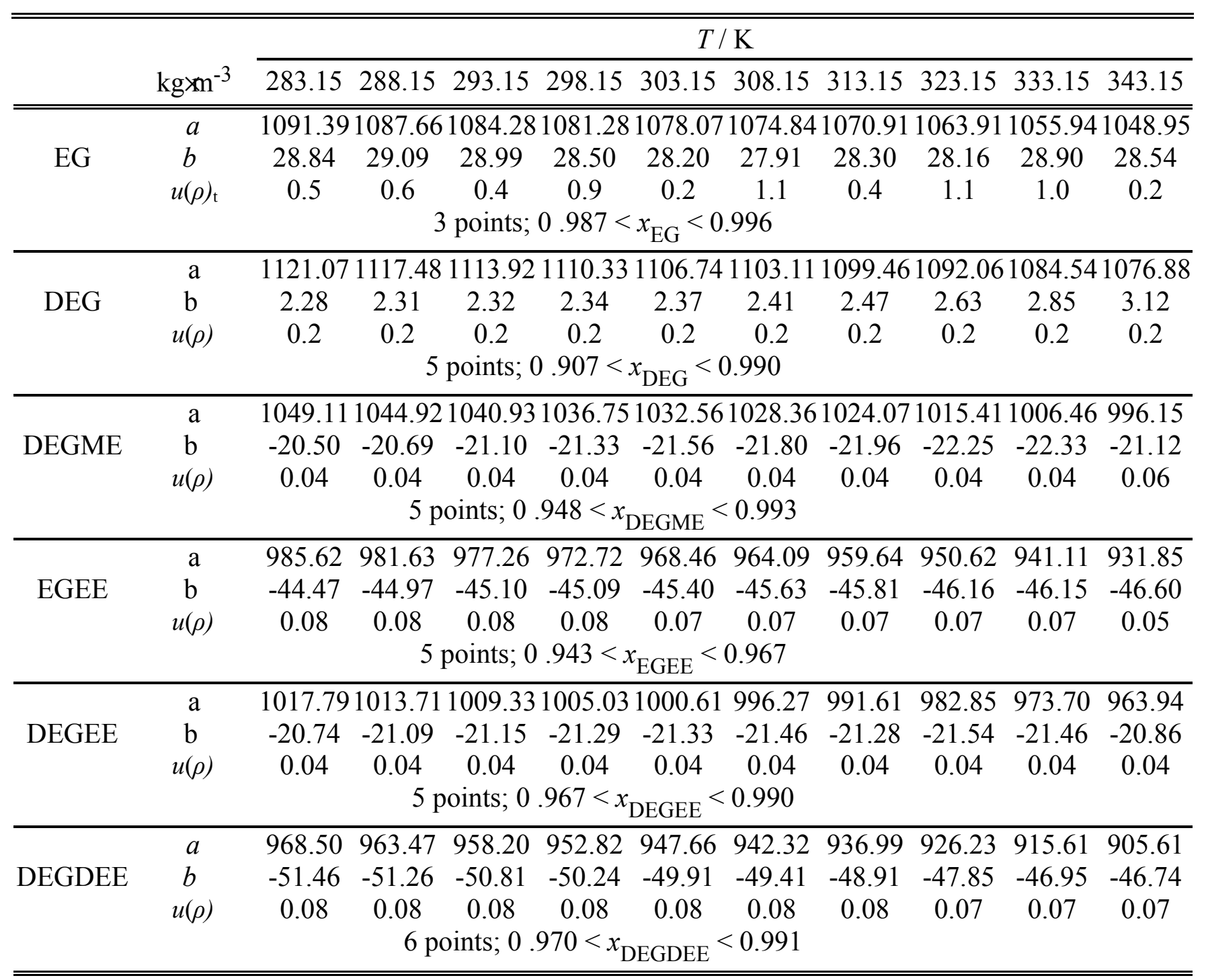


Table S4. Least squares fitting coefficients of the compound's aqueous solutions sound speed, uncertainties of the extrapolated sound speed of the pure glycols and glymes (calculated by error propagation), $u(u)$, the number of points used for each fit and the respective composition range.

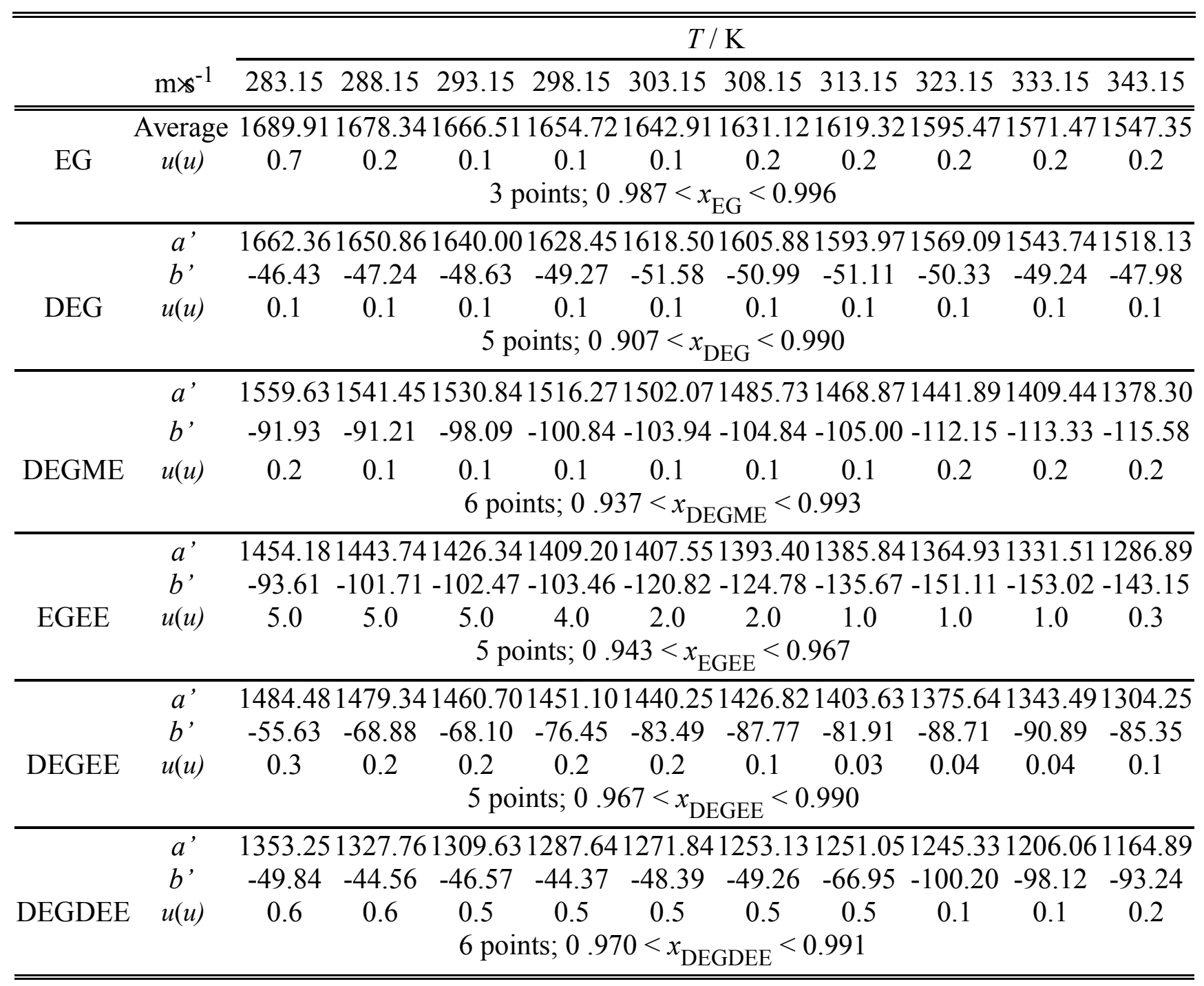


Table S5. Summary of experimental density, viscosity and sound speed data for the studied compounds, at atmospheric pressure.

\begin{tabular}{|c|c|c|c|c|c|}
\hline Reference & Compounds & Property & Method & $T_{\text {range }} / \mathbf{K}$ & $U()$ \\
\hline Albuquerque et al. ${ }^{11}$ & EG & $\rho ; n_{D}$ & VTD; RF & 298.15 & $0.00002 ; 0.00002$ \\
\hline Aminabhavi et al. ${ }^{25}$ & EG; DEG & $\rho ; \eta ; u ; n_{D}$ & PY; CV; I; RF & 298.15 & $0.0002 ; 0.001 ; 2 ; 0.0001$ \\
\hline Aminabhavi et al. ${ }^{36}$ & DEGDME & $\rho ; \eta ; u ; n_{D}$ & PY; CV; I; RF & 298 to 318 & $0.0002 ; 0.001 ; 2 ; 0.0001$ \\
\hline Aralaguppi et al. ${ }^{47}$ & EGEE & $\rho ; \eta ; u ; n_{D}$ & PY; CV; I; RF & 298 to 308 & $0.0002 ; 0.001 ; 2 ; 0.0002$ \\
\hline Awasthi et al. ${ }^{59}$ & EGEE & $\rho ; \eta$ & PY; CV & 303 to 323 & $0.00001 ; 0.0001$ \\
\hline Awwad et al. ${ }^{70}$ & $\mathrm{EG}$ & $\rho ; n_{D}$ & VTD; RF & 303.15 & $0.00003 ; 0.0002$ \\
\hline Begum et al. ${ }^{81}$ & TriEG; TeEG; TeEGDME & $\rho ; \eta ; \gamma$ & $\mathrm{PY} ; \mathrm{CV} ; \mathrm{WP}$ & 293 to 323 & $0.00015 ; 0.04 ; 0.51$ \\
\hline Bernal-Garcia et al. ${ }^{85}$ & DEG & $\rho ; \eta$ & VTD; CV & 283 to 343 & $0.00003 ; 0.004$ \\
\hline Chandrasekhar et al. ${ }^{1}$ & EGEE & $\rho ; u$ & PY; I & $298.15 ; 308.15$ & $0.00002 ; 0.2 \%$ \\
\hline Chiao and Thompson ${ }^{2}$ & DEGME; DEGEE & $\rho ; n_{D}$ & PY; RF & & $0.0001 ; 0.14 \%$ \\
\hline Chu and Thompson ${ }^{3}$ & EGEE & $\rho ; n_{D}$ & PY; RF & $293.15 ; 298.15$ & $0.0001 ; 0.0001$ \\
\hline Cocchi et al. ${ }^{4}$ & EG & $\rho$ & VTD & 273 to 313 & 0.00001 \\
\hline Comunas et al. ${ }^{5}$ & EGEE & $\rho$ & VTD & 293 to 353 & 0.0001 \\
\hline Conesa et al. ${ }^{6}$ & $\begin{array}{l}\text { EGDME; DEGDME; TriEGDME; } \\
\text { TeEGDME }\end{array}$ & $\rho ; \eta$ & VTD; CV & 283 to 423 & $0.00002 ; 0.0005$ \\
\hline Corradini et al. ${ }^{7}$ & EG & $\eta$ & $\mathrm{CV}$ & 283 to 353 & 0.004 \\
\hline Ćwiklińska et al. ${ }^{8}$ & EGEE; DEGEE & $\eta$ & $\mathrm{CV}$ & 293 to 303 & 0.001 \\
\hline Das et al. ${ }^{9}$ & EGEE & $\rho ; \eta ; u$ & PY; CV; I & 298.15 & $0.00003 ; 0.0002 ; 0.2$ \\
\hline
\end{tabular}




\begin{tabular}{|c|c|c|c|c|c|}
\hline de Ruiz Holgado et al. ${ }^{10}$ & DEGDME & $\rho ; \eta$ & VTD; CV & 298.15 & $0.0003 ; 0.005$ \\
\hline de Ruiz Holgado et al. ${ }^{12}$ & TeEGDME & $\rho ; \eta$ & VTD; CV & 278 to 318 & $0.0002 ; 0.0003$ \\
\hline Dhondge et al. ${ }^{13}$ & EGEE; DEGME; DEGEE & $\rho ; \eta$ & PY; RB & $275.15 ; 283.15$ & $0.00005 ; 1 \%$ \\
\hline Dhondge et al. ${ }^{14}$ & DEGDME & $\rho ; u ; n_{D}$ & PY; I; RF & 298.15 & $0.00005 ; 0.5 ; 0.0001$ \\
\hline Douhéret et al. ${ }^{15,16}$ & EGEE; DEGEE & $u$ & $\mathrm{I}$ & 298.15 & 0.1 \\
\hline Douhéret et al. ${ }^{17-19}$ & EG; DEG; TriEG; DEGME & $\rho ; u$ & VTD; I & 288 to 308 & $0.0005 ; 0.1$ \\
\hline Ge et al. ${ }^{20}$ & EG & $\rho ; \eta$ & PY; RB & 298 to 338 & $1 \% ; 1 \%$ \\
\hline George et al. ${ }^{21}$ & EG; DEG; TriEG; TeEG & $\rho ; u ; n_{D}$ & VTD; I; NA & 298 to 348 & $0.00001 ; 1.3 ; \mathrm{NA}$ \\
\hline Hartel et al. ${ }^{22}$ & TriEG; TriEGDME & $\rho ; \eta$ & $\mathrm{NA}$; NA & $283 ; 313$ to 353 & $\mathrm{NA}$; NA \\
\hline Henni et al. ${ }^{23}$ & TriEGDME & $\rho ; \eta$ & VTD; CV & 298 to 343 & $0.00005 ; 1 \%$ \\
\hline Henni et al. ${ }^{24}$ & TeEGDME & $\rho ; \eta$ & VTD; CV & 298 to 343 & $0.00005 ; 1 \%$ \\
\hline Iglesias-Otero et al. ${ }^{26}$ & EG & $\rho ; n_{D}$ & VTD; RF & 298.15 & $0.0001 ; 0.0002$ \\
\hline Islam et al. ${ }^{27}$ & EGEE & $\rho ; \eta$ & PY; CV & 303 to 323 & $0.00003 ; 0.5 \%$ \\
\hline Jerome et al. ${ }^{28}$ & EG; TriEG & $\eta$ & $\mathrm{CV}$ & 298.15 & $0.3 \%$ \\
\hline Jiménez et al. ${ }^{29}$ & EG & $\rho ; n_{D} ; \gamma$ & VTD; RF; PD & 293 to 308 & $0.002 ; 0.0001 ; 0.01$ \\
\hline Johnson et al. ${ }^{30}$ & EGEE & $\rho ; \eta ; u$ & PY; CV; I & 308.15 & $0.0001 ; 0.001 ; 1$ \\
\hline Kinart et al. ${ }^{31}$ & DEG; TriEG; TeEG & $\eta ; u$ & $\mathrm{CV} ; \mathrm{I}$ & 293 to 303 & $0.002 ; 0.01$ \\
\hline Kinart et al..$^{32}$ & DEGME & $\eta$ & $\mathrm{CV}$ & 293 to 303 & 0.001 \\
\hline Klimaszewski et al. ${ }^{33}$ & TeEG & $\rho ; u$ & VTD; I & 288 to 328 & $0.00001 ; 0.1$ \\
\hline
\end{tabular}




\begin{tabular}{|c|c|c|c|c|c|}
\hline Kodama et al. $^{34}$ & DEGDME; TriEGDME; TeEGDME & $\rho ; \eta$ & VTD; RB & 313.15 & $0.00005 ; 3 \%$ \\
\hline $\mathrm{Ku}$ et $\mathrm{al} .{ }^{35}$ & EGDME; DEGDME; DEGDEE & $\rho ; \eta ; n_{D}$ & VTD; CV; RF & 288 to 343 & $0.1 \% ; 0.9 \% ; 0.00001$ \\
\hline Ku et al. ${ }^{37}$ & $\begin{array}{l}\text { EGDME: DEGDME; TriEGDME; } \\
\text { TeEGDME }\end{array}$ & $\rho ; n_{D}$ & VTD; RF & 288 to 318 & $0.00002 ; 0.00002$ \\
\hline Kumagai et al. ${ }^{38}$ & EG; DEG; TriEG; TeEG & $\rho ; \eta$ & PY; FB; & 273 to 333 & $0.04 \% ; 0.7 \%$ \\
\hline Lee et al. ${ }^{39}$ & EG; DEG; TriEG; TeEG & $\eta$ & $\mathrm{CV}$ & 294 to 427 & $1.5 \%$ \\
\hline Li et al. ${ }^{40}$ & EG & $\rho$ & PY & 293 to 328 & 0.00003 \\
\hline Li et al. ${ }^{41}$ & DEGME & $\rho ; \eta$ & $\mathrm{PY} ; \mathrm{CV}$ & 293 to 333 & $0.1 \% ; 0.15 \%$ \\
\hline Lopez et al. ${ }^{42}$ & DEGME & $u$ & I & 293 to 353 & $0.2 \%$ \\
\hline Marchetti et al. ${ }^{43}$ & EG & $\rho ; \eta$ & VTD; CV & 293 to 353 & $0.000001 ; 0.004$ \\
\hline Marchetti et al. ${ }^{44}$ & EG & $n_{D}$ & $\mathrm{RF}$ & 263 to 353 & 0.0001 \\
\hline $\mathrm{McGee}^{45}$ & TeEGDME & $\rho ; \eta ; n_{D}$ & $\mathrm{PY} ; \mathrm{CV} ; \mathrm{RF}$ & 298.15 & $0.0009 ; \mathrm{NA} ; 0.0002$ \\
\hline Mesquita et al. ${ }^{46}$ & DEG: TriEG & $\rho ; \eta$ & VTD; RB & 298.15 & $0.0005 ; 0.35 \%$ \\
\hline Miller, K.J. ${ }^{48}$ & EGEE & $\rho ; \eta ; n_{D}$ & PY; CV; RF & 298.15 & $\mathrm{NA}$; NA; 0.0001 \\
\hline Mozo et al. ${ }^{49,50}$ & EGEE; DEGME & $\rho ; u$ & VTD; I & 293 to 303 & $0.000002 ; 0.3$ \\
\hline Nayak et al..$^{51}$ & EG & $\rho ; \eta ; u ; n_{D}$ & PY; CV; I; RF & 298 to 308 & $0.0001 ; 0.001 ; 2 ; 0.001$ \\
\hline Pal et al..$^{52}$ & EG; DEG; TriEG; TeEG & $\rho ; \eta ; u$ & PY; CV; I & $303.15 ; 308.15$ & $0.0001 ; 0.003 ; 0.2$ \\
\hline Pal et al. ${ }^{53}$ & EG; DEG & $\rho ; \eta$ & $\mathrm{PY} ; \mathrm{CV}$ & 298.15 & $0.00005 ; 0.003$ \\
\hline Pal et al..$^{54}$ & TriEGDME & $\rho ; \eta ; n_{D}$ & $\mathrm{PY} ; \mathrm{CV} ; \mathrm{RF}$ & 298.15 & $0.00005 ; 0.003 ; 0.0001$ \\
\hline
\end{tabular}




\begin{tabular}{|c|c|c|c|c|c|}
\hline Pal et al. ${ }^{55}$ & $\begin{array}{l}\text { EGDME; DEGDME; TriEGDME; } \\
\text { TeEGDME }\end{array}$ & $\rho ; \eta$ & PY; CV & 298.15 & $0.0003 ; 0.003$ \\
\hline Pal et al. ${ }^{56}$ & DEGDEE & $\rho ; \eta$ & PY; CV & 298 to 318 & $0.0001 ; 0.003$ \\
\hline Pal et al..$^{57}$ & DEGME; DEGEE & $\eta$ & $\mathrm{CV}$ & 298.15 & 0.003 \\
\hline Pal et al. ${ }^{58}$ & DEGME; DEGEE & $\rho ; u$ & PY; CV & 298.15 & $0.00003 ; 0.2$ \\
\hline Pandhurnekar et al. ${ }^{60}$ & EGEE; DEGME; DEGEE & $\rho ; u ; n_{D}$ & PY; I; RF & $278.15 ; 288.15$ & $0.00005 ; 0.5 ; 0.0001$ \\
\hline Pereira et al. ${ }^{61}$ & TriEGDME & $\rho ; u ; n_{D}$ & VTD; I; RF & 288 to 308 & $0.0001 ; 0.1 ; 0.00001$ \\
\hline Quijada et al. ${ }^{62}$ & EG & $\rho ; \eta$ & VTD; CV & 298 to 328 & $0.000005 ; 0.65 \%$ \\
\hline Real et al. ${ }^{63}$ & TeEGDME & $\rho ; u ; n_{D}$ & VTD; I; RF & 288 to 308 & $0.0001 ; 0.00005 ; 1$ \\
\hline Ren et al. ${ }^{64}$ & EG; DEG & $\rho ; \eta$ & $\mathrm{PY} ; \mathrm{CV}$ & 303 to 323 & $0.0002 ; 0.1 \%$ \\
\hline Rinkenbach et al. ${ }^{65}$ & DEG & $\rho ; \eta ; n_{D}$ & PY; CV; RF & 174 to 306 & NA; NA; NA \\
\hline Roy et al. ${ }^{66}$ & EGEE & $\rho ; \eta ; u ; n_{D}$ & PY; CV; I; RF & 303 to 323 & $0.0002 ; 0.0002 ; 0.8 ; 0.0002$ \\
\hline Sagdeev et al. ${ }^{67}$ & EG & $\rho ; \eta$ & HW; FB & 293 to 464 & $(0.15$ to 0.30$) \% ;(1.5$ to 2.0$) \%$ \\
\hline Sastry et al. ${ }^{68}$ & EG; DEG; TriEG & $\rho ; \eta ; u$ & VTD; CV; I & 298 to 313 & $0.00003 ; 0.003 ; 1.3$ \\
\hline Seikel et al. ${ }^{69}$ & DEGEE; DEGME & $\rho ; n_{D}$ & $\mathrm{NA}$; NA & $293.15 ; 298.15$ & NA; NA \\
\hline Sun et al..$^{71}$ & EG & $\rho ; \eta$ & PY; CV & 290 to 450 & $0.2 \% ; 2 \%$ \\
\hline Tamura et al. ${ }^{72}$ & EGEE & $\rho ; u$ & VTD; I & $298.15 ; 303.15$ & $0.00002 ; 0.1$ \\
\hline Tawfik et al. ${ }^{73}$ & EG & $\rho$ & VTD & 303 to 422 & 0.0002 \\
\hline Tsai et al. ${ }^{74}$ & TriEG & $\rho ; \eta$ & PY; CV & 303 to 343 & $0.05 \% ; 1 \%$ \\
\hline
\end{tabular}


Tsierkezos et al. ${ }^{77}$

EG; DEG; TriEG; TeEG

Wallace et al. ${ }^{78}$

Wallace et al. ${ }^{79}$

Yu et al. ${ }^{80}$

Zivkovic et al. ${ }^{82}$

Zorębski and Lubowiecka-Kostka ${ }^{83}$

Zorębski and Waligóra ${ }^{84}$ $\rho ; \eta ; n_{D} ; \gamma \quad$ VTD; CV; RF; DNR 283 to 313

$\begin{array}{lll}\rho ; u & \text { VTD; I } & 298.15 \\ \rho ; \eta ; n_{D} & \text { PY; CV; RF } & 298.15 \\ \rho ; \eta ; n_{D} & \text { PY } \text { CV } ; \text { RF } & 298.15\end{array}$

$\rho ; \eta ; n_{D}$

\section{RF}

$\rho ; \eta ; n_{D} \quad$ VTD; RB; RF

298.15

288 to 323

$\rho ; \eta ; u \quad$ VTD; CV; PEO

298 to 313

293 to 313

$\rho ; n_{D} \quad$ VTD; RF
$0.00001 ; 0.001 ; 0.0001 ; 0.1$

$0.00005 ; 1$

0.0005; NA; 0.0001

$\mathrm{NA}$; NA; NA

NA
$0.002 ; 0.8 \% ; 0.00006$

$0.00005 ; 0.2 \% ; 0.1$

\subsection{5; NA}

$\rho$ - density; $\eta$ - viscosity; $u$ - sound speed; $n_{D}$ - Refractive index; $\gamma$ - surface tension; VTD - vibrating tube densimeter; CV - Capillary viscometer; PYC pycnometer; HW - hydrostatic weighing; FB - falling-body method; RB - rotational viscometer; RF - refactrometer; I - ultrasonic interferometer; PEO - pulseecho-overlap method; DNR - Du Nouy ring; PD - pendant drop method; WP - Wilhelmy plate; NA - information not available.

combined expanded uncertainties units are; $U(\rho)$ in $\mathrm{g} . \mathrm{cm}^{-3}, U(\eta)$ in $\mathrm{mPa} . \mathrm{s}, U(u)$ in $\mathrm{m}_{\mathrm{s}} \mathrm{s}^{-1}, U\left(n_{D}\right)$ in $\mathrm{n}_{\mathrm{D}}$ and $U(\sigma)$ in $\mathrm{mN} . \mathrm{m}^{-1}$, except if other units are mentioned 
Table S6. Empirical correlations of the viscosity, sound speed and refractive index experimental data.

\begin{tabular}{ll}
\hline EG & DEG \\
$\eta=\exp [-2.3568+617.200 /(T-179.414)]$ & $\eta=\exp [-2.5622+723.618 /(\mathrm{T}-175.571)]$ \\
$n_{D}=\left(-3.0121 \cdot 10^{-4} \pm 6.5 \cdot 10^{-7}\right) \cdot T+\left(1.51990 \pm 2.0 \cdot 10^{-4}\right)$ & $n_{D}=\left(-3.1893 \cdot 10^{-4} \pm 3.7 \cdot 10^{-7}\right) \cdot T+\left(1.54050 \pm 1.2 \cdot 10^{-4}\right)$ \\
$u=\left(-2.3745 \pm 4.1 \cdot 10^{-3}\right) \cdot T+(2362.6 \pm 1.3)$ & $u=\left(-2.4259 \pm 2.7 \cdot 10^{-3}\right) \cdot T+(2302.6 \pm 0.8)$ \\
TriEG & TeEG \\
$\eta=\exp [-2.6357+787.734 /(\mathrm{T}-172.191)]$ & $\eta=\exp [-2.6238+818.977 /(\mathrm{T}-171.168)]$ \\
$n_{D}=\left(-3.3091 \cdot 10^{-4} \pm 5.4 \cdot 10^{-7}\right) \cdot T+\left(1.55313 \pm 1.7 \cdot 10^{-4}\right)$ & $n_{D}=\left(-3.4147 \cdot 10^{-4} \pm 5.0 \cdot 10^{-7}\right) \cdot T+\left(1.56008 \pm 1.6 \cdot 10^{-4}\right)$ \\
$u=\left(-3.015 \pm 1.5 \cdot 10^{-2}\right) \cdot T+(2510.9 \pm 4.5)$ & $u=\left(-3.130 \pm 2.0 \cdot 10^{-2}\right) \cdot T+(2531.7 \pm 6.2)$ \\
DEGDME & TriEGDME \\
$\eta=\exp [-2.328+335.020 /(T-154.903)]$ & $\eta=\exp [-2.266+409.375 /(T-159.307)]$ \\
$n_{D}=\left(-4.3731 \cdot 10^{-4} \pm 7.8 \cdot 10^{-7}\right) \cdot T+\left(1.53621 \pm 2.4 \cdot 10^{-4}\right)$ & $n_{D}=\left(-4.2669 \cdot 10^{-4} \pm 2.5 \cdot 10^{-7}\right) \cdot T+\left(1.547940 \pm 7.9 \cdot 10^{-5}\right)$ \\
$u=\left(-3.915 \pm 1.7 \cdot 10^{-2}\right) \cdot T+(2447.6 \pm 5.3)$ & $u=\left(-3.794 \pm 2.2 \cdot 10^{-2}\right) \cdot T+(2473.7 \pm 6.8)$ \\
TeEGDME & DEGDEE \\
$\eta=\exp [-2.310+484.727 /(T-161.306)]$ & $\eta=\exp [-2.590+421.682 /(\mathrm{T}-149.566)]$ \\
$n_{D}=\left(-4.047 \cdot 10^{-4} \pm 1.3 \cdot 10^{-6}\right) \cdot T+\left(1.55059 \pm 4.1 \cdot 10^{-4}\right)$ & $n_{D}=\left(-4.5488 \cdot 10^{-4} \pm 2.4 \cdot 10^{-7}\right) \cdot T+\left(1.545378 \pm 7.5 \cdot 10^{-5}\right)$ \\
$u=\left(-3.692 \pm 2.3 \cdot 10^{-2}\right) \cdot T+(2483.3 \pm 7.1)$ & $u=\left(-3.875 \pm 2.4 \cdot 10^{-2}\right) \cdot T+(2398.9 \pm 7.3)$ \\
DEGME & DEGEE \\
$\eta=\exp [-2.752+604.927 /(T-146.089)]$ & $\eta=\exp [-2.113+370.955 /(T-192.340)]$ \\
$n_{D}=\left(-3.819 \cdot 10^{-4} \pm 3.7 \cdot 10^{-6}\right) \cdot T+\left(1.5373 \pm 1.2 \cdot 10^{-3}\right)$ & $n_{D}=\left(-4.0538 \cdot 10^{-4} \pm 9.0 \cdot 10^{-7}\right) \cdot T+\left(1.54588 \pm 2.8 \cdot 10^{-4}\right)$ \\
$u=\left(-3.4181 \pm 9.7 \cdot 10^{-3}\right) \cdot T+(2434.7 \pm 3.0)$ & $u=\left(-3.500 \pm 1.5 \cdot 10^{-2}\right) \cdot T+(2418.4 \pm 4.7)$ \\
EGEE & \\
$\eta=\exp [-4.254+1108.435 /(\mathrm{T}-70.499)]$ & \\
$n_{D}=\left(-4.412 \cdot 10^{-4} \pm 2.4 \cdot 10^{-6}\right) \cdot T+\left(1.53970 \pm 7.5 \cdot 10^{-4}\right)$ & \\
$u=\left(-3.626 \pm 1.4 \cdot 10^{-2}\right) \cdot T+(2386.4 \pm 4.3)$ & \\
\hline
\end{tabular}


Table S7. Parameters of the least squares fitting equation of density as a function of temperature, for the 11 glycols studied, and the corresponding standard deviations of the fits. In parenthesis are the standard uncertainties affecting each parameter.

\begin{tabular}{|c|c|c|c|c|c|c|}
\hline & $A$ & $B$ & $C$ & $D$ & $E$ & $\sigma_{\text {fit }}$ \\
\hline & \multicolumn{6}{|c|}{$\left(\mathrm{kg} \times \mathrm{m}^{-3}\right)$} \\
\hline EG & $\begin{array}{l}1370.7 \\
(3.5)\end{array}$ & $\begin{array}{c}-1.3024 \\
(0.034)\end{array}$ & $\begin{array}{c}2.2794 \times 10^{-3} \\
\left(1.1 \times 10^{-4}\right)\end{array}$ & $\begin{array}{c}-2.8382 \times 10^{-6} \\
\left(1.2 \times 10^{-7}\right)\end{array}$ & - & 0.0019 \\
\hline DEG & $\begin{array}{c}1376.9 \\
(6.0)\end{array}$ & $\begin{array}{r}-1.2813 \\
(0.058)\end{array}$ & $\begin{array}{c}2.0720 \times 10^{-3} \\
\left(1.8 \times 10^{-4}\right)\end{array}$ & $\begin{array}{c}-2.5040 \times 10^{-6} \\
\left(2.0 \times 10^{-7}\right)\end{array}$ & - & 0.0032 \\
\hline $\operatorname{TrEG}$ & $\begin{array}{c}1629 \\
(40)\end{array}$ & $\begin{array}{c}-4.1528 \\
(0.52)\end{array}$ & $\begin{array}{c}1.5243 \times 10^{-2} \\
\left(2.5 \times 10^{-3}\right)\end{array}$ & $\begin{array}{c}-3.0263 \times 10^{-5} \\
\left(5.3 \times 10^{-6}\right)\end{array}$ & $\begin{array}{c}2.2227 \times 10^{-8} \\
\left(4.2 \times 10^{-9}\right)\end{array}$ & 0.0010 \\
\hline TeEG & $\begin{array}{c}1409 \\
(11)\end{array}$ & $\begin{array}{c}-1.2802 \\
(0.11)\end{array}$ & $\begin{array}{c}1.5143 \times 10^{-3} \\
\left(3.4 \times 10^{-4}\right)\end{array}$ & $\begin{array}{c}-1.5795 \times 10^{-6} \\
\left(3.6 \times 10^{-7}\right)\end{array}$ & - & 0.0059 \\
\hline DEGME & $\begin{array}{l}1554 \\
(41)\end{array}$ & $\begin{array}{c}-4.4764 \\
(0.52)\end{array}$ & $\begin{array}{c}1.7307 \times 10^{-2} \\
\left(2.5 \times 10^{-3}\right)\end{array}$ & $\begin{array}{c}-3.6394 \times 10^{-5} \\
\left(5.3 \times 10^{-6}\right)\end{array}$ & $\begin{array}{c}2.8101 \times 10^{-8} \\
\left(4.3 \times 10^{-9}\right)\end{array}$ & 0.0011 \\
\hline DEGDME & $\begin{array}{l}1236.0 \\
(4.4)\end{array}$ & $\begin{array}{r}-1.0851 \\
(0.042)\end{array}$ & $\begin{array}{c}5.8328 \times 10^{-4} \\
\left(1.4 \times 10^{-4}\right)\end{array}$ & $\begin{array}{c}-9.6528 \times 10^{-7} \\
\left(1.4 \times 10^{-7}\right)\end{array}$ & - & 0.0023 \\
\hline TrEGDME & $\begin{array}{l}1280.0 \\
(3.3)\end{array}$ & $\begin{array}{r}-1.1323 \\
(0.032)\end{array}$ & $\begin{array}{c}6.5458 \times 10^{-4} \\
\left(1.0 \times 10^{-4}\right)\end{array}$ & $\begin{array}{c}-7.8131 \times 10^{-7} \\
\left(1.1 \times 10^{-7}\right)\end{array}$ & - & 0.0018 \\
\hline TeEGDME & $\begin{array}{c}1305.9 \\
(2.1)\end{array}$ & $\begin{array}{r}-1.1323 \\
(0.020)\end{array}$ & $\begin{array}{c}6.0857 \times 10^{-4} \\
\left(6.5 \times 10^{-5}\right)\end{array}$ & $\begin{array}{c}-5.8733 \times 10^{-7} \\
\left(6.9 \times 10^{-8}\right)\end{array}$ & - & 0.0011 \\
\hline EGEE & $\begin{array}{l}1224 \\
(13)\end{array}$ & $\begin{array}{c}-1.3305 \\
(0.12)\end{array}$ & $\begin{array}{c}1.9889 \times 10^{-3} \\
\left(4.0 \times 10^{-4}\right)\end{array}$ & $\begin{array}{c}-2.8695 \times 10^{-6} \\
\left(4.2 \times 10^{-7}\right)\end{array}$ & - & 0.0068 \\
\hline DEGEE & $\begin{array}{c}1272.7 \\
(6.9)\end{array}$ & $\begin{array}{c}-1.1914 \\
(0.066)\end{array}$ & $\begin{array}{c}1.2262 \times 10^{-3} \\
\left(2.1 \times 10^{-4}\right)\end{array}$ & $\begin{array}{c}-1.6127 \times 10^{-6} \\
\left(2.3 \times 10^{-7}\right)\end{array}$ & - & 0.0037 \\
\hline DEGDEE & $\begin{array}{l}1232.3 \\
(3.8)\end{array}$ & $\begin{array}{c}-1.4049 \\
(0.036)\end{array}$ & $\begin{array}{c}1.5343 \times 10^{-3} \\
\left(1.2 \times 10^{-4}\right)\end{array}$ & $\begin{array}{c}-1.7813 \times 10^{-6} \\
\left(1.2 \times 10^{-7}\right)\end{array}$ & - & 0.0020 \\
\hline
\end{tabular}




\section{References}

(1) Chandrasekhar, G.; Venkatesu, P.; Venkata, M.; Rao, P. Excess Molar Volumes and Speed of Sound of Ethyl Acetate and Butyl Acetate with 2-Alkoxyethanols at 308.15 K. Engineering 2000, No. 1, 590593.

(2) Chiao, T.-. Densities and Refractive Indices of Diethylene Glycol Ether-Water Solutions. Diethylene Glycol Monomethyl, Monoethyl, and Monobutyl Ethers. J. Chem. Eng. Data 1961, 6, 192-193.

(3) Chu, K. Y.; Thompson, A. R. Densities and Refractive Indices of Glycol Ether-Water Solutions. J. Chem. Eng. Data 1960, 5, 147-149.

(4) Cocchi, M.; Marchetti, A.; Pigani, L.; Sanna, G.; Tassi, L.; Ulrici, A.; Vaccari, G.; Zanardi, C. Density and Volumetric Properties of Ethane-1,2-Diol+di-Ethylen-Glycol Mixtures at Different Temperatures. Fluid Phase Equilib. 2000, 172, 93-104.

(5) Comuñas, M. J. P.; Reghem, P.; Baylaucq, A.; Boned, C.; Fernández, J. High-Pressure Volumetric Properties of Three Monoethylene Glycol Alkyl Ethers. J. Chem. Eng. Data 2004, 49, 1344-1349.

(6) Conesa, A.; Shen, S.; Coronas, A. Liquid Densities, Kinematic Viscosities, and Heat Capacities of Some Ethylene Glycol Dimethyl Ethers at Temperatures from 283.15 to $423.15 \mathrm{~K}$. Int. J. Thermophys. 1998, 19, 1343-1358.

(7) Corradini, F.; Franchini, G.; Marchetti, A.; Tagliazucchi, M.; Tassi, L.; Tosi, G. Viscosities of 1,2Ethanediol-2-Methoxyethanol Solvent Mixtures at Various Temperatures. J. Solution Chem. 1993, 22, $1019-1028$.

(8) Ćwiklińska, A.; Dzikowski, T.; Szychowski, D.; Kinart, W. J.; Kinart, C. M. Viscosities of Binary Mixtures of Some N-Ethoxyethanols with Ethyl Tert-Butyl Ether at $\mathrm{T}=(293.15,298.15$, and 303.15)K. J. Chem. Thermodyn. 2007, 39, 1325-1330.

(9) Das, M.; Roy, M. N. Volumetric, Viscosimetric and Acoustic Studies of Binary Mixtures of 2Ethoxyethanol with 1-Alkanols at 298.15 K. Phys. Chem. Liq. 2006, 44, 663-685.

(10) de Ruiz Holgado, M. E.; de Schaefer, C. R.; Arancibia, E. L.; Katz, M. Excess Molar Volumes and Viscosities of Binary Mixtures of bis(2-Methoxyl)ether (diglyme) with Chloroalkanes at $298.15 \mathrm{~K}$. Fluid Phase Equilib. 1994, 95, 299-312.

(11) Albuquerque, L.; Ventura, C.; Gonçalves, R. Refractive Indices, Densities, and Excess Properties for Binary Mixtures Containing Methanol, Ethanol, 1,2-Ethanediol, and 2-Methoxyethanol. J. Chem. Eng. Data 1996, 41, 685-688.

(12) Ruiz Holgado, M. E. F. de; Schaefer, C. R. De; Arancibia, E. L. Densities and Viscosities of Binary Mixtures of Polyethylene Glycol 350 Monomethyl Ether with N -Butanol and N -Pentanol and Tetraethylene Glycol Dimethyl Ethers with N -Propanol, N -Butanol, and N -Pentanol from 278.15 K to 318.15 K. J. Chem. Eng. Data 2002, 47, 144-148.

(13) Dhondge, S. S.; Pandhurnekar, C. P.; Sheikh, S.; Deshmukh, D. W. Volumetric and Viscometric Study of Aqueous Binary Mixtures of Some Glycol Ethers at $\mathrm{T}=(275.15$ and 283.15$) \mathrm{K}$. J. Chem. Thermodyn. 2011, 43, 1961-1968.

(14) Dhondge, S. S.; Pandhurnekar, C. P.; Parwate, D. V. Density, Speed of Sound, and Refractive Index of Aqueous Binary Mixtures of Some Glycol Ethers at T $=298.15$ K. J. Chem. Eng. Data 2010, 55, 3962-3968.

(15) Douhéret, G.; Pal, A.; Davis, M. I. Ultrasonic Speeds and Isentropic Functions of (a 2-Alkoxyethanol + Water) at 298.15 K. J. Chem. Thermodyn. 1990, 22, 99-108.

(16) Douhéret, G.; Salgado, C.; Davis, M. I.; Loya, J. Ultrasonic Speeds and Isentropic Functions of 2-(2Alkoxyethoxy)ethanol + Water at 298.15 K. Thermochim. Acta 1992, 207, 313-328.

(17) Douheret, G.; Pal, A.; Høiland, H.; Anowi, O.; Davis, M. I. Thermodynamic Properties of (ethan-1,2Diol + Water) at the Temperature 298.15 K I. Molar Volumes, Isobaric Molar Heat Capacities, Ultrasonic Speeds, and Isentropic Functions. J. Chem. Thermodyn. 1991, 23, 569-580.

(18) Douhéret, G.; Davis, M. I.; Ulloa, J.; Hoiland, H.; Fjellanger, I. J. Ultrasonic Speeds and Volumetric Properties of Binary Mixtures of Water with 2-[2-(2-Alkoxyethoxy)ethoxy]ethanols at $298.15 \mathrm{~K} . J$. Chem. Soc. Faraday Trans. 1996, 92, 2369.

(19) Douhéret, G.; Lajoie, P.; Davis, M. I.; Ratliff, J. L.; Ulloa, J.; Hoiland, H. Volumetric Properties of Binary Mixtures of Water with Methoxy(ethoxy) N Ethanols. J. Chem. Soc. Faraday Trans. 1995, 91, 2291.

(20) Ge, M.; Ma, J.; Chu, B. Densities and Viscosities of Propane-1,2,3-Triol + Ethane-1,2-Diol at T = (298.15 to 338.15) K. J. Chem. Eng. Data 2010, 55, 2649-2651. 
(21) George, J.; Sastry, N. V. Partial Excess Molar Volumes, Partial Excess Isentropic Compressibilities and Relative Permittivities of Water + Ethane-1,2-Diol Derivative and Water + 1,2-Dimethoxyethane at Different Temperatures. Fluid Phase Equilib. 2004, 216, 307-321.

(22) Haertel, G. H. Low-Volatility Polar Organic Solvents for Sulfur Dioxide Hydrogen Sulfide and Carbonyl Sulfide. J. Chem. Eng. Data 1985, 30, 57-61.

(23) Henni, A.; Naami, A.; Tontiwachwuthikul, P. Densities, Viscosities, and Derived Functions of Binary Mixtures : ( Triethylene Glycol Dimethyl Ether + Water ) and ( N-Acetylmorpholine + Water ) from 298.15 K to 343.15 K. J. Chem. Eng. Data 2005, 50, 1038-1042.

(24) Henni, A.; Naami, A.; Tontiwachwuthikul, P. Densities, Viscosities, and Derived Functions of Binary Mixtures: (Triethylene Glycol Dimethyl Ether + Water) and ( N -Acetylmorpholine + Water) from 298.15 K to 343.15 K. J. Chem. Eng. Data 2005, 50, 1038-1042.

(25) Aminabhavi, T. M.; Gopalakrishna, B. Density, Viscosity, Refractive Index, and Speed of Sound in Aqueous Mixtures of N,N-Dimethylformamide, Dimethyl Sulfoxide, N,N-Dimethylacetamide, Acetonitrile, Ethylene Glycol, Diethylene Glycol, 1,4-Dioxane, Tetrahydrofuran, 2-Methoxyethanol, and 2-Ethox. J. Chem. Eng. Data 1995, 40, 856-861.

(26) Iglesias-Otero, M. A.; Troncoso, J.; Carballo, E.; Romaní, L. Density and Refractive Index in Mixtures of Ionic Liquids and Organic Solvents: Correlations and Predictions. J. Chem. Thermodyn. 2008, 40, 949-956.

(27) Islam, M. N.; Islam, M. M.; Yeasmin, M. N. Viscosity of Aqueous Solutions of 2-Methoxyethanol, 2Ethoxyethanol, and Ethanolamine. J. Chem. Thermodyn. 2004, 36, 889-893.

(28) Jerome, F. S.; Tseng, J. T.; Fan, L. T. Viscosities of Aqueous Glycol Solutions. J. Chem. Eng. Data 1968, 13, 496-496.

(29) Jiménez, E.; Cabanas, M.; Segade, L.; García-Garabal, S.; Casas, H. Excess Volume, Changes of Refractive Index and Surface Tension of Binary 1,2-Ethanediol + 1-Propanol or 1-Butanol Mixtures at Several Temperatures. Fluid Phase Equilib. 2001, 180, 151-164.

(30) Johnson, I.; Kalidoss, M.; Srinivasamoorthy, R. Density, Viscosity, and Speed of Sound in the Ternary Mixtures of 2-Ethoxyethanol $+\mathrm{N}, \mathrm{N}$-Dimethylformamide $+\mathrm{N}, \mathrm{N}$-Dimethylacetamide and 2Ethoxyethanol + Dimethyl Sulfoxide $+\mathrm{N}, \mathrm{N}$-Dimethylacetamide at 308.15 K. J. Chem. Eng. Data 2002, 47, 1388-1390.

(31) Kinart, C. M.; Klimczak, M.; Ćwiklińska, A. Viscosities, Speeds of Sound and Isentropic Compressibilities for Binary Mixtures of 2-Propoxyethanol with Ethylene Glycols. J. Mol. Liq. 2010, $155,127-133$.

(32) Kinart, C. M.; Ćwiklińska, A.; Kinart, W. J.; Bald, A. Viscosities of Some N-Alkoxyethanols with Ethyl Tert-Butyl Ether at T=(293.15, 298.15 and 303.15) K. J. Mol. Liq. 2006, 126, 135-139.

(33) Klimaszewski, K.; Stronka-Lewkowska, E.; Trzcińska, I.; Bald, A. Volumetric and Acoustic Studies on (tetraethylene Glycol+water) Mixtures in a Wide Temperature Range. J. Chem. Thermodyn. 2015, $85,191-201$.

(34) Kodama, D.; Kanakubo, M.; Kokubo, M.; Hashimoto, S.; Nanjo, H.; Kato, M. Density, Viscosity, and Solubility of Carbon Dioxide in Glymes. Fluid Phase Equilib. 2011, 302, 103-108.

(35) Ku, H.; Tu, C. Densities and Viscosities of Seven Glycol Ethers from 288.15 K to 343.15 K. J. Chem. Eng. Data 2000, 45, 391-394.

(36) Aminabhavi, T. M.; Phayde, H. T. S.; Khinnavar, R. S.; Gopalakrishna, B.; Hansen, K. C. Densities, Refractive Indices, Speeds of Sound, and Shear Viscosities of Diethylene Glycol Dimethyl Ether with Ethyl Acetate, Methyl Benzoate, Ethyl Benzoate, and Diethyl Succinate in the Temperature Range from 298.15 to 318.15 K. J. Chem. Eng. Data 1994, 39, 251-260.

(37) Ku, S. C.; Tu, C. H. Liquid Densities and Refractive Indices of Binary Mixtures for the Dimethyl Ether of a Glycol + Ethanol from T $=288.15 \mathrm{~K}$ to 318.15 K. J. Chem. Eng. Data 2004, 49, 357-362.

(38) Kumagai, A.; Mochida, H.; Takahashi, S. Liquid Viscosities and Densities of HFC-134a+glycol Mixtures. Int. J. Thermophys. 1993, 14, 45-53.

(39) Lee, R. J.; Teja, A. S. Viscosities of Poly(ethylene Glycols). J. Chem. Eng. Data 1990, 35, 385-387.

(40) Li, Q.; Tian, Y.; Wang, S. Densities and Excess Molar Volumes for Binary Mixtures of 1,4-Butanediol + 1,2-Propanediol, + 1,3-Propanediol, and + Ethane-1,2-Diol from (293.15 to 328.15) K. J. Chem. Eng. Data 2008, 53, 271-274.

(41) Li, X.-X.; Zhou, W.-D.; Li, X.-Y.; Sun, J.-L.; Jiang, W. Density, Viscosity, and Excess Properties of the Binary Mixture of Diethylene Glycol Monomethyl Ether+water at T=(293.15, 303.15, 313.15, 323.15, 333.15) K under Atmospheric Pressure. J. Mol. Liq. 2009, 148, 73-76.

(42) López, E. R.; Daridon, J. L.; Plantier, F.; Boned, C.; Fernández, J. Temperature and Pressure 
Dependences of Thermophysical Properties of Some Ethylene Glycol Dimethyl Ethers from Ultrasonic Measurements. Int. J. Thermophys. 2006, 27, 1354-1372.

(43) Marchetti, A.; Preti, C.; Tagliazucchi, M.; Tassi, L.; Tosi, G. The N,N-Dimethylformamide/ethane1,2-Diol Solvent System. Density, Viscosity, and Excess Molar Volume at Various Temperatures. $J$. Chem. Eng. Data 1991, 36, 360-365.

(44) Marchetti, A.; Preti, C.; Tagliazucchi, M.; Tassi, L.; Tosi, G. The N,N-Dimethylformamide + Ethane1,2-Diol Solvent System. Dielectric Constant, Refractive Index, and Related Properties at Various Temperatures. J. Chem. Eng. Data 1991, 36, 365-368.

(45) McGee, R. L.; Wallace, W. J.; Rataiczak, R. D. Densities, Viscosities, Refractive Indexes, and Molar Refractions of the Binary System Tetraethylene Glycol Dimethyl Ether-Water at 25.degree.C. J. Chem. Eng. Data 1983, 28, 305-307.

(46) Mesquita, F. M. R.; Feitosa, F. X.; Aznar, M.; de Sant'Ana, H. B.; Santiago-Aguiar, R. S. Density, Viscosities, and Excess Properties for Binary Mixtures of Sulfolane + Alcohols and Sulfolane + Glycols at Different Temperatures. J. Chem. Eng. Data 2014, 59, 2196-2206.

(47) Aralaguppi, M. I.; Jadar, C. V; Aminabhavi, T. M. Density, Refractive Index, Viscosity, and Speed of Sound in Binary Mixtures of 2-Ethoxyethanol with Dioxane, Acetonitrile, and Tetrahydrofuran at (298.15, 303.15, and 308.15) K. J. Chem. Eng. Data 1996, 41, 1307-1310.

(48) Miller, K. J. Some Physical Properties of the System 2-Ethoxyethanol-Butyl Acetate. J. Phys. Chem. 1958, 62, 512-512.

(49) Mozo, I.; González, J. A.; García de la Fuente, I.; Cobos, J. C.; Riesco, N. Thermodynamics of Mixtures Containing Alkoxyethanols. Part XXV. Densities, Excess Molar Volumes and Speeds of Sound at 293.15, 298.15 and $303.15 \mathrm{~K}$, and Isothermal Compressibilities at $298.15 \mathrm{~K}$ for 2alkoxyethanol+1-Butanol Systems. J. Mol. Liq. 2008, 140, 87-100.

(50) Mozo, I.; García de la Fuente, I.; González, J. A.; Cobos, J. C.; Riesco, N. Thermodynamics of Mixtures Containing Alkoxyethanols. Part XXVI. Densities, Excess Molar Volumes, Speeds of Sound at $(293.15,298.15$, and 303.15) K, and Isentropic or Isothermal Compressibilities at $298.15 \mathrm{~K}$ for 2Methoxyethanol + Alkoxyethanol or 2-Propo. J. Chem. Eng. Data 2008, 53, 1404-1410.

(51) Nayak, J. N.; Aralaguppi, M. I.; Aminabhavi, T. M. Density, Viscosity, Refractive Index, and Speed of Sound in the Binary Mixtures of 1,4-Dioxane + Ethanediol, + Hexane, + Tributylamine, or + Triethylamine at $(298.15,303.15$, and 308.15) K. J. Chem. Eng. Data 2003, 48, 1152-1156.

(52) Pal, A.; Singh, W. Speeds of Sound and Viscosities in Aqueous Poly(ethylene Glycol) Solutions at 303.15 and 308.15 K. J. Chem. Eng. Data 1997, 42, 234-237.

(53) Pal, A.; Sharma, S. Excess Molar Volumes and Viscosities of 1-Propanol + Ethylene Glycol, + Ethylene Glycol Monomethyl, + Ethylene Glycol Dimethyl, + Diethylene Glycol Dimethyl, + Triethylene Glycol Dimethyl, + Diethylene Glycol Diethyl, and + Diethylene Glycol Dibutyl Ether. $J$. Chem. Eng. Data 1998, 43, 532-536.

(54) Pal, A.; Dass, G.; Kumar, A. Excess Molar Volumes, Viscosities, and Refractive Indices of Triethylene Glycol Dimethyl Ether with Dimethyl Carbonate, Diethyl Carbonate, and Propylene Carbonate at 298.15 K. J. Chem. Eng. Data 1998, 43, 738-741.

(55) Pal, A.; Singh, Y. P. Viscosity in Water + Ethylene Glycol Dimethyl, +Diethylene Glycol Dimethyl, +Triethylene Glycol Dimethyl, and +Tetraethylene Glycol Dimethyl Ethers at 298.15 K. J. Chem. Eng. Data 1996, 41, 1008-1011.

(56) Pal, A.; Dass, G. Excess Molar Volumes and Viscosities of Diethylene Glycol Diethyl Ether with Dimethyl Carbonate, Diethyl Carbonate, and Propylene Carbonate at $(298.15,308.15$, and 318.15) K. J. Chem. Eng. Data 2000, 45, 487-491.

(57) Pal, A.; Kumar, H. Viscosities for Binary Liquid Mixtures of Some N-Alkoxyethanols with Alkyl Acetates at 298.15 K. Indian J. Chem. -Section A 2000, 39A, 1322-1326.

(58) Pal, A.; Kumar, A.; Kumar, H. Speeds of Sound and Isentropic Compressibilities of NAlkoxyethanols and Polyethers with Propylamine at 298.15 K. Int. J. Thermophys. 2006, 27, $777-$ 793.

(59) Awasthi, A.; Awasthi, A. Intermolecular Interactions in formamide+2-Alkoxyethanols: Viscometric Study. Thermochim. Acta 2012, 537, 57-64.

(60) Pandhurnekar, C. P.; Parwate, D. V.; Dhondge, S. S. Investigations of Volumetric, Acoustic and Optical Properties of Aqueous Binary Mixtures of Different Glycol Ethers at Temperatures $278.15 \mathrm{~K}$ and 288.15 K. J. Mol. Liq. 2013, 183, 94-101.

(61) Pereira, S. M.; Rivas, M. A.; Real, J. N.; Legido, J. L.; Iglesias, T. P. Densities, Speeds of Sound, and Refractive Indices of the Mixture Nonane + Triethylene Glycol Dimethyl Ether at 288.15 K, 293.15 
K, 298.15 K, and 308.15 K. J. Chem. Eng. Data 2002, 47, 919-922.

(62) Quijada-Maldonado, E.; Meindersma, G. W.; de Haan, A. B. Viscosity and Density Data for the Ternary System water(1)-ethanol(2)-ethylene glycol(3) between $298.15 \mathrm{~K}$ and 328.15K. J. Chem. Thermodyn. 2012, 57, 500-505.

(63) Real, J. N.; Iglesias, T. P.; Pereira, S. M.; Rivas, M. A. Analysis of Temperature Dependence of Some Physical Properties of (n-Nonane+ Tetraethylene Glycol Dimethyl Ether). J. Chem. Thermodyn. 2002, 34, 1029-1043.

(64) Ren, D.-H.; Fang, S.; Xu, X.-J.; Ding, B. Volumetric Properties and Viscosities of Acetic Acid With Ethylene Glycol and Diethylene Glycol At Temperatures From 303.15 To 323.15 K. Chem. Eng. Commun. 2014, 201, 528-544.

(65) Rinkenbach, W. H. Properties of Diethylene Glycol 1. Ind. Eng. Chem. 1927, 19, 474-476.

(66) Roy, M. N.; Sah, R. S.; Pradhan, P. Densities, Viscosities, Sound Speeds, Refractive Indices, and Excess Properties of Binary Mixtures of Isoamyl Alcohol with Some Alkoxyethanols. Int. J. Thermophys. 2010, 31, 316-326.

(67) Sagdeev, D. I.; Fomina, M. G.; Mukhamedzyanov, G. K.; Abdulagatov, I. M. Experimental Study of the Density and Viscosity of Polyethylene Glycols and Their Mixtures at Temperatures from $293 \mathrm{~K}$ to $473 \mathrm{~K}$ and at Atmospheric Pressure. J. Chem. Thermodyn. 2011, 43, 1824-1843.

(68) Sastry, N. V.; Patel, M. C. Densities, Excess Molar Volumes, Viscosities, Speeds of Sound, Excess Isentropic Compressibilities, and Relative Permittivities for Alkyl (methyl, Ethyl, Butyl, and Isoamyl) Acetates + Glycols at Different Temperatures. J. Chem. Eng. Data 2003, 48, 1019-1027.

(69) Seikel, M. Ethylene Glycol Determination in and Removal from Commercial Alkyl Ethers of Diethylene Glycol. Ind. Eng. Chem. Anal. Ed. 1941, 13, 388-389.

(70) Awwad, A. M.; Al-Dujaili, A. H.; Salman, H. E. Relative Permittivities, Densities, and Refractive Indices of the Binary Mixtures of Sulfolane with Ethylene Glycol, Diethylene Glycol, and Poly(ethylene Glycol) at 303.15 K. J. Chem. Eng. Data 2002, 47, 421-424.

(71) Sun, T.; Teja, A. S. Density, Viscosity and Thermal Conductivity of Aqueous Solutions of Propylene Glycol, Dipropylene Glycol, and Tripropylene Glycol between 290 K and 460 K. J. Chem. Eng. Data 2004, 49, 1311-1317.

(72) Tamura, K.; Osaki, A.; Murakami, S.; Laurent, B.; Grolier, J. E. Thermodynamic Properties of Binary Mixtures $\{x(2$-alkoxyethanol $)+(1-\mathrm{x}) \mathrm{n}$-Octane $\}$ : Densities at 298.15 and $303.15 \mathrm{~K}$ and Speeds of Sound at 298.15 K. Fluid Phase Equilib. 2000, 173, 285-296.

(73) Tawfik, W. Y.; Teja, A. S. The Densities of Polyethylene Glycols. Chem. Eng. Sci. 1989, 44, 921-923.

(74) Tsai, C. Y.; Soriano, A. N.; Li, M. H. Vapour Pressures, Densities, and Viscosities of the Aqueous Solutions Containing (triethylene Glycol or Propylene Glycol) and ( $\mathrm{LiCl}$ or $\mathrm{LiBr}$ ). J. Chem. Thermodyn. 2009, 41, 623-631.

(75) Tseng, Y.-M.; Thompson, A. R. Communication. Densities and Refractive Indices of Diethylene Glycol Ether-Water Solutions. J. Chem. Eng. Data 1962, 7, 483-483.

(76) Tsierkezos, N. G.; Molinou, I. E. Thermodynamic Properties of Water + Ethylene Glycol at 283.15, 293.15, 303.15, and 313.15 K. J. Chem. Eng. Data 1998, 43, 989-993.

(77) Tsierkezos, N. G.; Palaiologou, M. M. Ultrasonic Studies of Liquid Mixtures of Either Water or Dimethylsulfoxide with Ethylene Glycol, Diethylene Glycol, Triethylene Glycol, Tetraethylene Glycol, 1,2-Propylene Glycol and 1,4-Butylene Glycol at 298.15 K. Phys. Chem. Liq. 2009, 47, 447459.

(78) Wallace, W. J.; Shephard, C. S.; Underwood, C. Densities, Refractive Indexes, Molar Refractions, Viscosities, and Dielectric Constants of Triethylene Glycol Dimethyl Ether-Water Solutions at 25.degree. J. Chem. Eng. Data 1968, 13, 11-13.

(79) Wallace, W. J.; Mathews, A. L. Density, Refractive Indices, Molar Refractions, and Viscosities of Diethylene Glycol Dimethyl Ether-Water Solutions at 25o C. J. Chem. Eng. Data 1964, 9, 267-268.

(80) Yu, Y.-X.; Liu, J.-G.; Gao, G.-H. Isobaric Vapor-liquid Equilibria of Three Aromatic HydrocarbonTetraethylene Glycol Binary Systems. Fluid Phase Equilib. 1999, 157, 299-307.

(81) Begum, S. K.; Clarke, R. J.; Ahmed, M. S.; Begum, S.; Saleh, M. a. Volumetric, Viscosimetric and Surface Properties of Aqueous Solutions of Triethylene Glycol, Tetraethylene Glycol, and Tetraethylene Glycol Dimethyl Ether. J. Mol. Liq. 2013, 177, 11-18.

(82) Živković, E. M.; Bajić, D. M.; Radović, I. R.; Šerbanović, S. P.; Kijevčanin, M. L. Volumetric and Viscometric Behavior of the Binary Systems Ethyl lactate+1,2-Propanediol, +1,3-Propanediol, +tetrahydrofuran and +tetraethylene Glycol Dimethyl Ether. New UNIFAC-VISCO and ASOGVISCO Parameters Determination. Fluid Phase Equilib. 2014, 373, 1-19. 
(83) Zorębski, E.; Lubowiecka-Kostka, B. Thermodynamic and Transport Properties of (1,2-ethanediol+1Nonanol) at Temperatures from (298.15 to 313.15)K. J. Chem. Thermodyn. 2009, 41, 197-204.

(84) Zorębski, E.; Waligóra, A. Densities, Excess Molar Volumes, and Isobaric Thermal Expansibilities for 1,2-Ethanediol + 1-Butanol, or 1-Hexanol, or 1-Octanol in the Temperature Range from (293.15 to 313.15) K. J. Chem. Eng. Data 2008, 53, 591-595.

(85) Manuel Bernal-García, J.; Guzmán-López, A.; Cabrales-Torres, A.; Rico-Ramírez, V.; Iglesias-Silva, G. A. Supplementary Densities and Viscosities of Aqueous Solutions of Diethylene Glycol from (283.15 to 353.15) K. J. Chem. Eng. Data 2008, 53, 1028-1031. 